\title{
NONLINEAR EVOLUTION OF THE PARKER INSTABILITY
}

\author{
R. MATSUMOTO \\ College of Arts and Sciences, Chiba University, \\ Yayoi-cho, Chiba 260, Japan \\ K. SHIBATA \\ Department of Earth Sciences, Aichi University of Education, \\ Kariya, Aichi 448, Japan
}

\begin{abstract}
Two-dimensional MHD simulations are performed to study the nonlinear evolution of the Parker instability in galactic gas disks. When the most unstable mode grows, magnetic field lines kink across the equatorial plane of the disk and thin spur-like structures are formed above dense regions in magnetic pockets. In low $\beta\left(=p_{\text {gas }} / p_{\text {mag }}<3\right)$ disks, shock waves are produced at the footpoint of magnetic loops, while in high $\beta(>3)$ disks, nonlinear oscillations are excited and the loop length increases with time up to $\lambda_{c} \simeq(3.5 \beta+6) H$, where $H$ is the half-thickness of the disk.
\end{abstract}

\section{INTRODUCTION}

It is suggested that the Parker instability is related to the formation of interstellar cloud complexes (Parker 1966; Mouschovias et al. 1974) and the Radio spurs and HI spurs observed in our galaxy (Sofue 1973,1976). Although the linear stage of the Parker instability has been studied extensively (e.g., Parker 1966), the nonlinear stage of this instability is not well understood. Thus we performed two-dimensional MHD simulations to study its nonlinear evolution (Matsumoto et al. 1988,1990; Shibata et al. 1990).

\section{NUMERICAL MODEL}

We consider a local part of galactic gas disks in a Cartesian geometry $(x, z)$, where $x$-axis is parallel to the azimuthal direction and $z$-axis is perpendicular to the disk. For simplicity, we assume a plane parallel gas layer located at a distance $R$ from a point mass $M$ which is the origin of the gravity, and neglect effects of cosmic ray pressure, rotation, and self-gravity of the gas disk. Thus the gravitational acceleration in $z$-direction is $g(z)=G M z /\left(R^{2}+z^{2}\right)^{3 / 2}$. Magnetic fields are assumed to be 
initially horizontal in magnetostatic equilibrium. Two kinds of initial magnetized disk (and halo) are studied: (1) the isothermal gas layer with constant Alfvén speed (i.e., the magnetic flux is not localized), (2) the magnetized disk with a hot halo (magnetic flux is localized around the equatorial plane). Parameters describing the equilibrium model are $\beta=p_{\text {gas }} / p_{\text {mag }}$ and $\epsilon=G M /\left[(1+1 / \beta) C_{s}^{2} R\right]$, where the plasma $\beta$ and the sound speed $C_{s}$ are evaluated at the equatorial plane. Note that $p(z=\infty) / p(z=0)=\exp (-\epsilon)$ for isothermal disk with constant $\beta$. Thus the external (or halo) pressure is large for small $\epsilon$ disk.

\section{RESULTS OF NONLINEAR NUMERICAL SIMULATIONS}

\subsection{Spurs and Shock Waves}

Figure 1 shows the fully nonlinear stage of the Parker instability in the isothermal gas disk with initially constant Alfvén speed $(\beta=1$ and $\epsilon=6)$. As the instability develops for the most unstable mode, magnetic field lines kink across the equatorial plane of the disk and dense regions are created in the pockets of magnetic loops. Above these dense regions, spur-like structures perpendicular to the disk plane are formed. These spurs explain thermal spurs observed in our galaxy (e.g., Müller et al. 1987). At the footpoint of magnetic loops, shock waves are formed because the speed of downflow along magnetic loops is comparable to the initial Alfvén speed (= $1.4 C_{s}$ in this model) and is larger than the sound speed. The configuration of these shock wave fronts is similar to the distribution of molecular clouds in the Orion region (e.g., Maddalena et al. 1986). We propose that shock waves produced by the Parker instability trigger the formation of molecular clouds. After the stage shown in Figure 1, expansion of magnetic loops is almost stopped owing to the high external pressure. The region behind shock waves settles into a quasi-static state.

\subsection{The Length of Magnetic Loops}

Figure 2 shows the results of parameter survey for the isothermal disk. Here, the wavelength of perturbation $(\lambda)$ is normalized by the local density scale height $H$. In low $\beta(<3)$ disks, shock waves are formed when the most unstable perturbation grows. Owing to the energy dissipation due to the shock, the system quickly settles into the quasi-static state where the loop length is equal to the most unstable wavelength. On the other hand, in high $\beta(>3)$ disks, nonlinear oscillations are excited and the loop length gradually increases with time owing to the mode coupling. The final length of the loop is determined by the condition of shock wave formation (the upper hatched region in Figure 2), and is $\lambda_{c} \simeq(3.5 \beta+6) H$. Therefore, the loop length in the high $\beta$ disk is much longer than the most unstable wavelength. A long $(\sim 4.5 \mathrm{kpc})$ arc-like structure of the magnetic field observed in M31 (Beck and Berkhuijsen 1990) may correspond to our long loop ( $\sim 4 \mathrm{kpc})$ for $\beta \simeq 10$. 

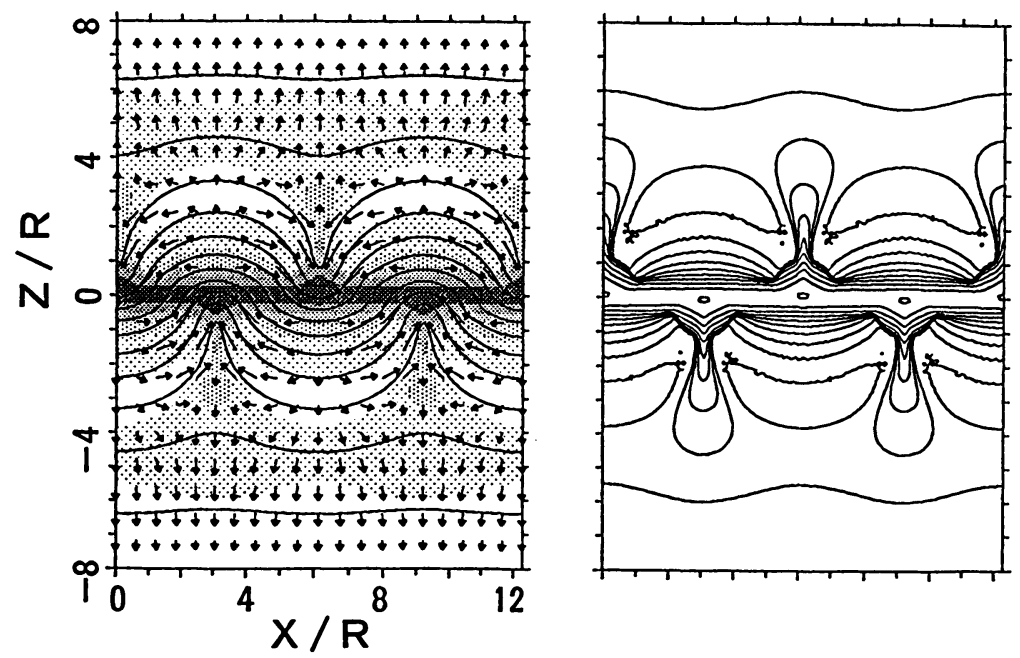

Figure 1. Nonlinear stage of the Parker instability in the isothermal disk with $\beta=1$ and $\epsilon=6$. The left panel shows the density distribution (grey scale), velocity field (arrows), and magnetic field lines (solid curves) at $t=8.55 R / C_{s}$. The right panel shows the density contours. The contour level step width is $\Delta \log \rho=0.25$. The region where the contour lines are close each other represent shock wave fronts.

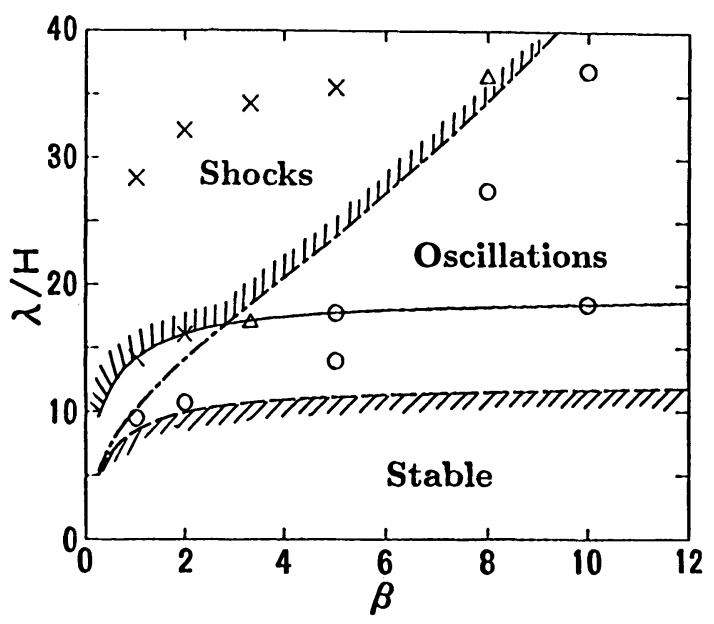

Figure 2. Criterion of shock wave formation and nonlinear oscillation for the isothermal disk with $\epsilon=6$. Crosses show the cases where shock waves are formed. Circles denote the cases where the system shows nonlinear oscillations. Triangles are intermediate cases. Solid curve shows the linearly most unstable wavelength. Upper hatched area denotes the expected size of quasi-static magnetic loops. 

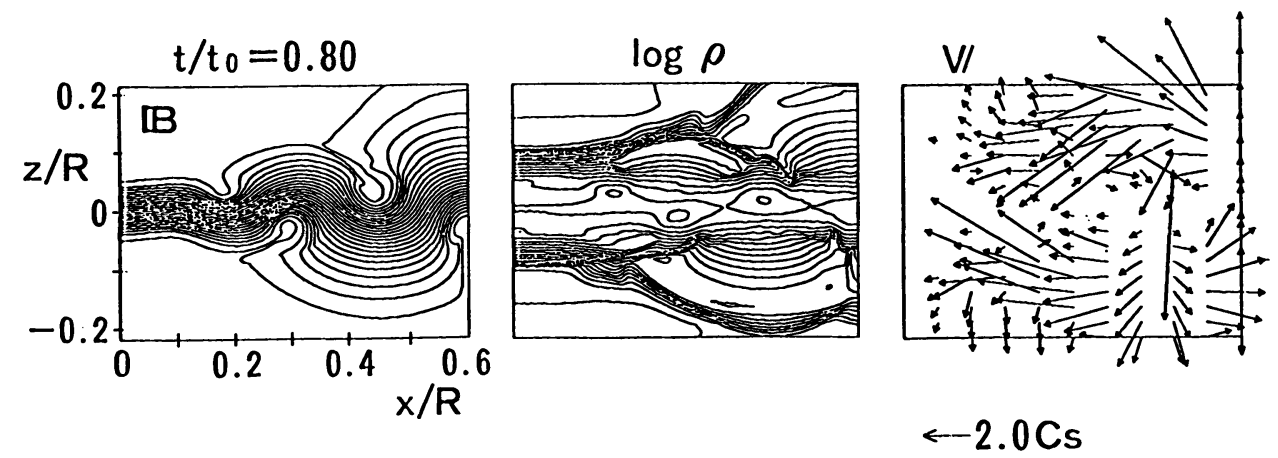

Figure 3. Numerical results of the Parker instability of a localized magnetic flux sheet.

\subsection{Self-Similar Expansion of Magnetic Loops}

Figure 3 shows the results when the magnetic flux is initially localized around the equatorial plane. In the initial equilibrium model, hot halo $\left(T_{\text {halo }} / T_{\text {disk }}=100\right)$ exists in $|z|>0.1 R$. Other parameters are $\beta=1$ and $\epsilon=1000$. Symmetric boundary condition is used at $x=0$ and $x=0.6 R$, and the imposed perturbation is localized in $0.45 R<x$. In this low halo pressure $\left(p_{\text {halo }} / p_{\text {disk }}<10^{-3}\right)$ model, magnetic loops continue to expand into the halo as long as the magnetic pressure in the loop is larger than the external halo pressure. The expansion of the magnetic loop is self-similar and the expansion speed increases linearly with height. These results are similar to the results of two-dimensional MHD simulations of emerging magnetic flux from the solar photosphere (Shibata et al. 1989). Figure 3 also shows that the Parker instability produces various structures such as dense blobs, magnetic loops, shock waves, neutral sheets, and spurs.

\section{REFERENCES}

Beck, R., Berkhuijsen, E.M. (1990) Proc. IAU Symp. No. 140, "Galactic and Intergalactic Magnetic Fields", eds. R. Beck, P. Kronberg, R. Wielebinski, Reidel, p. 198

Maddalena, R.J., Morris, M., Moscowitz J., Thaddeus, P. (1986) Ap. J. 303, 375

Matsumoto, R., Horiuchi, T., Shibata, K., Hanawa, T. (1988) Pub. Astr. Soc. Japan 40, 171

Matsumoto, R., Horiuchi, T., Hanawa, T., Shibata, K. (1990) Ap. J. 356, 259

Mouschovias, T.Ch., Shu, F.H., and Woodward, P.R. (1974) Astr. Ap. 33, 73

Müller, P., Reif, K., Reich, W. (1987) Astr. Ap. 183, 327

Parker, E.N., (1966) Ap. J.. 145, 811

Shibata, K., Tajima, T., Matsumoto, R., Horiuchi, T., Hanawa, T., Rosner, R., Uchida, Y. (1989) Ap. J. 338, 471

Shibata, K., Tajima, T., Matsumoto, R. (1990) Ap. J. 350, 295

Sofue, Y. (1973) Pub. Astr. Soc. Japan 25, 207

Sofue, Y. (1976) Astr. Ap. 48, 1 